\title{
Safety and efficacy of tipranavir co-administered with low-dose ritonavir in patients with advanced HIV-I infection and limited treatment options
}

\author{
S Esser*1, A Moll², V Cairns' ${ }^{3}$, H Jaeger ${ }^{4}$, S Mauss ${ }^{5}$, J van Lunzen ${ }^{6}$ and \\ J Goldbach 7
}

Address: ${ }^{1}$ University Hospital Essen, Essen, Germany, ${ }^{2} \mathrm{HIV}$ specialised practice, Berlin, Germany, ${ }^{3}$ Consultant statistician, Oxford, UK, ${ }^{4} \mathrm{HIV}$ specialised practice, Munich, Germany, ${ }^{5} \mathrm{HIV}$ specialised practice, Duesseldorf, Germany, ${ }^{6}$ University Medical Center, Hamburg-Eppendorf, Germany and ${ }^{7}$ Boehringer Ingelheim Pharma GmbH \& Co. KG, Ingelheim, Germany

* Corresponding author

from Ninth International Congress on Drug Therapy in HIV Infection

Glasgow, UK. 9-13 November 2008

Published: 10 November 2008

Journal of the International AIDS Society 2008, I I (SuppI I):P4I doi:I0.II86/I758-2652-II-SI-P4 I

This abstract is available from: http://www.jiasociety.org/content/II/SI/P4I

(C) 2008 Esser et al; licensee BioMed Central Ltd.

\section{Purpose of the study}

To assess the safety and efficacy of tipranavir co-administered with low-dose ritonavir (TPV/r) in clinical settings.

\section{Methods}

Data from the German open-label study (EAP). To be included in the study, adult patients (pts) had to be triple antiretroviral (ARV) class experienced having failed at least two previous PI-based regimens. TPV/r 500/200 mg, twice daily, was added to a background regimen chosen by the treating physician. All adverse events (AE) were reported regardless of causal relationship and degree of seriousness.

\section{Summary of results}

Data of 254 HIV-1 infected pts (median age 44 years; 229 males, 25 females) from 70 centres were available for analysis. Most pts were in advanced stages: CDC B3 (28\%) and C3 (57.1\%). Hepatitis B and C co-infection was reported for $12.6 \%$ and $3.9 \%$ of the pts, respectively. Median baseline HIV-RNA (VL) was $4.7 \log 10$ copies/mL and median CD4157 cells/ $\mu \mathrm{L}$. After 12 months of TPV/r treatment, median change from baseline in VL was -1.9 $\log 10$ copies/mL and $+71.5 \mathrm{CD} 4$ cells $/ \mu \mathrm{L}$. Median TPV/r exposure was 35.4 weeks (1.1-76). 117 (46.1\%) pts experienced AEs considered related to TPV/r; 53 (20.9\%) pts developed serious AEs; 47 (18.5\%) pts discontinued TPV/ $\mathrm{r}$ due to AEs. Most commonly reported AEs in 33.5\% of pts were gastrointestinal disorders, the majority of which were mild or moderate intensity. In regard to ALT and AST elevations, $4.3 \%$ and $2 \%$ of pts had a maximum Grade 3 and $3.1 \%$ and $0.2 \%$ of pts had maximum Grade 4 elevation, respectively. No cases of jaundice or liver failure were reported.

\section{Conclusion}

Patients treated in the TPV EAP had advanced HIV-1 infection and were heavily pretreated. Nevertheless, they show remarkable decrease in VL and increase in $\mathrm{CD} 4$ cells/ $\mu \mathrm{L}$ The analysis of AEs did not reveal any new safety findings or change in the known AE profile of TPV/r. Notably, in this real world treatment setting, the rate of TPV/r discontinuations due to AEs was relatively low. 